TITLE:

\title{
Viscoelastic effects in relaxation processes of concentration fluctuations in dynamically asymmetric polymer blends
}

\section{$\operatorname{AUTHOR}(\mathrm{S}):$}

Takenaka, M; Takeno, H; Hasegawa, H; Saito, S; Hashimoto, T; Nagao, M

\section{CITATION:}

Takenaka, M ... [et al]. Viscoelastic effects in relaxation processes of concentration fluctuations in dynamically asymmetric polymer blends. Physical Review E 2002, 65(2): 021806.

\section{ISSUE DATE:}

2002-02

URL:

http://hdl.handle.net/2433/39902

RIGHT:

Copyright 2002 American Physical Society 


\title{
Viscoelastic effects in relaxation processes of concentration fluctuations in dynamically asymmetric polymer blends
}

\author{
Mikihito Takenaka, ${ }^{1}$ Hiroyuki Takeno, ${ }^{1}$ Hirokazu Hasegawa, ${ }^{1}$ Shin Saito, ${ }^{1}$ Takeji Hashimoto, ${ }^{1}$ and Michihiro Nagao ${ }^{2}$ \\ ${ }^{1}$ Department of Polymer Chemistry, Graduate School of Engineering, Kyoto University, Kyoto 606-8501, Japan \\ ${ }^{2}$ Institute for Solid State Physics, The University of Tokyo, Ibaraki 319-1106, Japan
}

(Received 12 July 2001; published 22 January 2002)

\begin{abstract}
Relaxation processes of the concentration fluctuations induced by a rapid pressure change were investigated for a dynamically asymmetric polymer blend [deuterated polybutadiene (DPB)/polyisoprene (PI)] with a composition of 50-50 by weight by using time-resolved small-angle neutron scattering. The pressure change was carried out inside the single-phase of the blend with the cell designed for polymeric systems under high pressure and temperature. Time change in the scattered intensity distribution with wave number $(q)$ during the relaxation processes was found to be approximated by Cahn-Hilliard-Cook linearized theory. The theoretical analysis yielded the $q$ dependence of Onsager kinetic coefficient that is characterized by the $q^{-2}$ dependence at $q \xi_{\mathrm{ve}}>1$ with the characteristic length $\xi_{\mathrm{ve}}$ (with $\xi_{\mathrm{ve}}$ being the viscoelastic length) being much larger than radius of gyration of DPB or PI. The estimated $\xi_{\mathrm{ve}}$ agrees well with that calculated using the Doi and Onuki theory that takes into account the viscoelastic effects arising from the dynamical asymmetry between the component polymers in the relaxation of concentration fluctuations.
\end{abstract}

DOI: 10.1103/PhysRevE.65.021806

PACS number(s): 46.35.+z

\section{INTRODUCTION}

The dynamics of concentration fluctuations in binary polymer blends has been one of the interesting research problems in chemical physics of complex liquids. Many experimental studies of the phase-separation processes of polymer blends have unveiled some common features between simple-liquid mixtures and polymer blends [1-4]. For example, the phase-separated structures of polymer blends and simple-liquids mixtures grow with dynamical self-similarity in the late stage spinodal decomposition (dynamical scaling concept [5-7]), and the coarsening behaviors of the two systems, as observed by time evolution of the characteristic wave number and scattered intensity at different quenches, become universal, independent of the quench depth and the systems when the relevant physical quantities are reduced with the quench-depth-dependent characteristic parameters (Langer-Bar-on-Miller's scaling postulate [8,9]). Those common features described above are mainly found with experiments using light scattering or optical microscope experiments, where the observed length scale $\ell$ and time scale $t$ are very much larger than the radius of gyration $\left(R_{g}\right)$ and characteristic time (the longest relaxation time $\tau_{m}[10,11]$ ) of polymers.

If we observe the dynamics in polymer blends at the length scale close to $R_{g}$ by using small-angle neutron scattering (SANS) or small-angle x-ray scattering (SAXS), some unique features, which are not relevant to small-molecular systems, appear in the dynamics of phase-separation processes. This is because linear flexible polymers have many internal degrees of freedom and hence internal modes of vibrations $[10,11]$. These modes should affect the dynamics at the length scale of the observation $\ell$ satisfying $\ell \leqslant R_{g}$ or at the time scale $t$ shorter than $\tau_{m}[10,11]$. One unique feature related to this is theoretically explored by Akcasu [12]: the phase-separation processes are affected by internal modes [12] at $\ell \leqslant R_{g}$ or at $t \leqslant \tau_{m}$, while they are affected by the self-diffusion of each component at $\ell \geqslant R_{g}$ or at $t \geqslant \tau_{m}$. The effects of internal modes on the dynamics of polymer blends have been experimentally studied in polystyrene (PS)/ poly(styrene-ran-4-bromostyrene) blend by Strobl [13] and in dPS/PS blend by Müller et al. [14].

Even in the case when $\ell \geqslant R_{g}$ or $t \geqslant \tau_{m}$, if $t$ and $\ell$ are not very large compared with $\tau_{m}$ and $R_{g}$, respectively, the normal modes still affect the dynamics through wave-number (q)-dependent translational diffusion via reptation [15]. This would make the Onsager kinetic coefficient $\Lambda$ nonlocal and hence $q$ dependent $[\Lambda=\Lambda(q)]$, which is not observed in other systems such as simple-liquid mixtures. It is expected that the Onsager kinetic coefficient asymptotically increases to a constant value $\Lambda(0)$ with $q R_{g} \rightarrow 0$. de Gennes [16] and Pincus [17] and later Binder [18] theoretically elucidated this effect. Pincus [17] predicted that the Onsager kinetic coefficients obeys $q^{-2}$ at $q R_{\Lambda} \geqslant 1$ with $R_{\Lambda}$ being $R_{g}$ for "symmetric" polymer blends (we will show the exact expression of the theory in Sec. III). According to the theory, $\Lambda(q)$ becomes effectively constant, satisfying $\Lambda(q) / \Lambda(0) \geqslant 0.95$, when $q R_{g} \leqslant 0.33$ for the symmetric blends. Here the symmetric blends denote that each component in polymer blends has an identical polymerization index $N$ and self-diffusion coefficient $D_{s}$.

Several experimental works investigated the $q$ dependence of the Onsager kinetic coefficient by analyzing the dynamics of early stage spinodal decomposition in the context of Pincus's [17] and Binder's [18] theory. Jinnai et al. [19] investigated the dynamics of the early stage spinodal decomposition of the nearly symmetric deuterated polybutadiene (DPB)/polybutadiene (PB) blend and determined the $q$ dependence of the Onsager kinetic coefficients. According to their study, the $q$ dependence of the Onsager kinetic coeffi- 
cient is well expressed by the Pincus theory but the estimated $R_{\Lambda}$ is about twice as large as $R_{g}$ 's of DPB or PB. Müller et al. [14] also found the $R_{\Lambda} / R_{g}=2$ for the nearly symmetric $\mathrm{dPS} / \mathrm{PS}$ blend. In both cases if the theory is correct, $R_{\Lambda}$ should be equal to $R_{g}$.

As for asymmetric blends in which each component in blends has different $D_{s}$ 's, Schwahn, Janssen, and Springer [20] found the $R_{\Lambda} / R_{g}$ is about 5 to 7 on the basis of the analysis described above for the dPS/poly(vinyl methyl ether) (PVME) blend. Kawasaki and Koga [21] suggested that this large $R_{\Lambda}$ originates from the dynamical coupling between diffusion and stress that is predicted as "viscoelastic effects" by Doi and Onuki [22,23] (DO). According to the DO theory, the stress relaxation governs the dynamics of the concentration fluctuations at $\ell$ shorter than a certain characteristic length $\xi_{\text {ve }}$ defined as "viscoelastic length," and the Onsager kinetic coefficient has $q^{-2}$ dependence at $q \xi_{\mathrm{ve}}>1$. $\xi_{\text {ve }}$ increases with asymmetry in polymerization indices and self-diffusion coefficients of the component polymers and can be much larger than $R_{g}$ and $R_{\Lambda}$.

The aim of this paper is to explore the effects of the dynamical coupling between diffusion and stress on the relaxation processes of the concentration fluctuations of dynamically asymmetric polymer blends within their one phase region. We focus on the long time relaxation processes where the Onsager kinetic coefficient should be a $q$-independent constant value $\Lambda(0)$, if the viscoelastic effects do not play an important role. In order to test the validity of the DO theory, we will estimate the $q$ dependence of the Onsager kinetic coefficient by analyzing the relaxation process with CahnHilliard-Cook (CHC) theory $[24,25]$ and compare the experimental value of $\xi_{\mathrm{ve}}$ with that estimated by the DO theory.

The blend samples and the experimental techniques used in this study are described in Sec. II. We will first show equilibrium structure factors for the blend in a single-phase state at a given temperature as a function of pressure and then characterize the pressure changes employed in this study in order to induce the relaxation of the concentration fluctuations in Sec. III A. In Sec. III B, we will present the experimental results on the time changes in the structure factors during the relaxation processes of the concentration fluctuations induced by the pressure change and analyze the data by using the CHC theory. We will discuss the viscoelastic effects on the $q$ dependence of the Onsager kinetic coefficient estimated by the $\mathrm{CHC}$ theory in Sec. III C. The Onsager kinetic coefficient thus evaluated will be compared with those predicted by the theories in Sec. III D. In Sec. III E, we will compare the viscoelastic length $\xi_{\text {ve-exp }}$ obtained from the relaxation experiments with that $\xi_{\text {ve-theory }}$, estimated by using the DO theory. The parameters required for estimating $\xi_{\text {ve-theory }}$ were obtained from viscoelastic data. Finally, we will summarize our results in Sec. IV.

\section{EXPERIMENTAL SECTION}

The DPB and polyisoprene (PI) used in this study were synthesized by living anionic polymerization. The characterization of DPB and PI are listed in Table I, where $M_{n}$ and $M_{w}$ designate number-averaged and weight-averaged mo-
TABLE I. Characterization of polymers used in this study

\begin{tabular}{ccccc}
\hline \hline Sample & $M_{w}$ (units & & $\begin{array}{c}\text { Molar volume } \\
\text { of monomer unit } \\
\left.\text { of } 10^{-4}\right)\end{array}$ & $\begin{array}{c}\text { Unperturbed } \\
\text { radius of } \\
\text { gyration }\end{array}$ \\
\hline DPB & $37.4^{\mathrm{a}}$ & $1.28^{\mathrm{a}}$ & 60.4 & ${\left.\mathrm{~cm}^{3} / \mathrm{mol}\right)}_{g}(\mathrm{~mm})$ \\
PI & $8.5^{\mathrm{b}}$ & $1.10^{\mathrm{b}}$ & 75.6 & 9.5 \\
\hline \hline
\end{tabular}

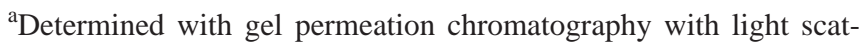
tering.

${ }^{b}$ Determined with gel permeation chromatography calibrated by polyisoprene standard samples.

${ }^{\mathrm{c}} v=M_{0} / \rho$, where $M_{0}$ and $\rho$ are, respectively, molecular weight of monomer and density $\left(0.899 \mathrm{~g} / \mathrm{cm}^{3}\right.$ for PI and $0.985 \mathrm{~g} / \mathrm{cm}^{3}$ for $\mathrm{DPB})$.

${ }^{\mathrm{d}} R_{g}$ are obtained with unperturbed chain parameters from the viscoelastic measurement [40]. We used the unperturbed chain parameters of polybutadiene to estimate $R_{g}$ of DPB.

lecular weight, respectively. The two polymers have different molecular weight: molecular weight ratio and molar volume ratio of DPB and PI are 4.40 and 4.42, respectively. The composition of a DPB/PI mixture studied was $50-50$ by weight. The DBP/PI blend has a lower-critical-solutiontemperature-type phase diagram and the spinodal temperature at $P=0.1 \mathrm{MPa}$ is $314.2 \mathrm{~K}$.

The DPB/PI blend was dissolved into a homogeneous solution with toluene in which total weight fraction of the polymers are 0.1 . The film specimen of the blend was obtained by evaporating the solvent slowly at room temperature. The film was further dried in vacuum for $24 \mathrm{~h}$ at room temperature, and then molded into the disk with $1-\mathrm{cm}$ diameter and 2-mm thickness for the SANS experiment. We installed the molded sample into the cell that is specially designed for the SANS measurement under high pressure (up to $200 \mathrm{MPa}$ ) and high temperature (up to $523 \mathrm{~K}$ ). The details of the cell for high pressure and temperature are described elsewhere [26]. The SANS experiments were performed with SANS-U of the Institute for Solid State Physics of the University of Tokyo at JRR-3M reactor at Japan Atomic Energy Research Institute in Tokai. The neutron wavelength $\lambda$ used here was 0.8 and $0.72 \mathrm{~nm}$ for the experiment at 298.1 and at $309.0 \mathrm{~K}$, respectively, but the sample-to-detector distance was fixed to $12 \mathrm{~m}$. All measured intensities were circularly averaged and calibrated into the absolute intensity $\left(\mathrm{cm}^{-1}\right.$ unit) with the incoherent scattering for Lupolene ${ }^{\circledR}$ after the correction of the electrical background noise, the sample transmittance, the scattering of an empty cell.

We measured the pressure dependence of the SANS intensity at $T=298.1$ and $309.0 \mathrm{~K}$ in order to investigate the pressure dependence of the Flory-Huggins segmental interaction parameter $\chi$ between DPB and PI. The pressure $P$ used here is $0.1,20.0,40.0,60.0$, and $80.0 \mathrm{MPa}$ at 298.1 and $0.1,20.0,40.0,60.0$, and $100.0 \mathrm{MPa}$ at $309.0 \mathrm{~K}$. For this purpose, SANS scattered intensity distribution was measured for $30 \mathrm{~min}$ at each temperature and at each pressure.

We took the following procedure to measure the time changes in the scattered intensity distribution induced by 
pressure changes (hereafter defined as "quench") from 80.0 to $0.1 \mathrm{MPa}$ at $298.1 \mathrm{~K}$ and from 100.0 to $0.1 \mathrm{MPa}$ at 309.0 $\mathrm{K}$ : for example, in the former quench, the sample was first equilibrated at $298.1 \mathrm{~K}$ and $80.0 \mathrm{MPa}$ for $30 \mathrm{~min}$ before the SANS measurement. The sample was then quenched from 80.0 to $0.1 \mathrm{MPa}$ at $298.1 \mathrm{~K}$, followed by a time-resolved SANS measurement as a function of time $t$ after the completion of the quench. The data acquisition during the relaxation process was implemented at 50 different time slices, each slice being obtained for data-acquisition time of $20 \mathrm{~s}$. Since each time-sliced scattered intensity distribution $S_{j}\left(q, t_{i}\right)$, thus obtained had a poor statistical accuracy, for a further quantitative analysis of it, we repeated the quench experiment over 20 times in order to obtain a signal-average scattered intensity distribution $S\left(q, t_{i}\right)$

$$
S\left(q, t_{i}\right)=\frac{1}{N} \sum_{j=1}^{N} S_{j}\left(q, t_{i}\right)
$$

where $N$ is the number of the repeated relaxation experiments $(N=20$ in this experiment), subscript $j$ denotes $j$ th relaxation experiment, and $t_{i}$ is the $i$ th time slice in each relaxation experiment. In order to avoid some artifacts involved by the signal-average process in each relaxation experiment, we confirmed the identity of the equilibrium scattered intensity distributions at 80.0 and $0.1 \mathrm{MPa}$ at 298.1 K. The same experimental procedure was taken for the quench from 100.0 to $0.1 \mathrm{MPa}$ at $309.0 \mathrm{~K}$.

In order to obtain the viscosity of the blend and the selfdiffusion coefficient of each component, we carried out linear viscoelastic measurements of the DPB/PI blend in its one phase region by using RMS-800, Rheometrics ${ }^{\circledR}$ with a coneplate geometry of diameter equal to $7.90 \mathrm{~mm}$ and angular frequency $\omega$ ranging from 0.002 to $100 \mathrm{rad} / \mathrm{sec}$. The strain amplitude used here is $2.0 \%$. The method to evaluate both quantities from the linear viscoelasticity will be detailed later in Sec. III D.

\section{RESULTS AND DISCUSSIONS}

\section{A. Quench depth induced by pressure jump}

Figures 1 and 2 show the pressure $(P)$ dependence of the equilibrium scattering function $S_{\mathrm{eq}}(q)$ at $298.1 \mathrm{~K}$ and 309.0 $\mathrm{K}$, respectively. In the figures, $S_{\mathrm{eq}}(q)$ is plotted as a function of wave number $q$ defined by

$$
q=(4 \pi / \lambda) \sin (\theta / 2)
$$

where $\theta$ is the scattering angle. $S_{\text {eq }}(q)$ decreases with pressure, indicating that the DPB/PI mixture has an uppercritical-solution-pressure-type phase diagram [27].

According to the scattering theory based on the random phase approximation (RPA) [28-30], $S_{\text {eq }}(q)$ is expressed by

$$
\frac{k_{N}}{S_{\mathrm{eq}}(q)}=\frac{1}{\phi_{\mathrm{DPB}} v_{\mathrm{DPB}} S_{\mathrm{DPB}}(q)}+\frac{1}{\phi_{\mathrm{PI}} v_{\mathrm{PI}} S_{\mathrm{PI}}(q)}-\frac{2 \chi}{v_{0}}
$$

with

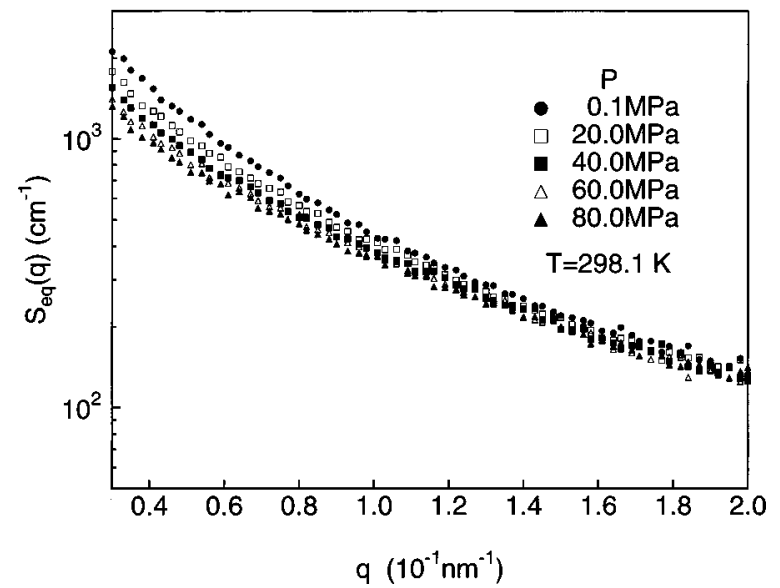

FIG. 1. Pressure dependence of the equilibrium scattering function $S_{\text {eq }}(q)$ at $298.1 \mathrm{~K}$ plotted as a function of $q$.

$$
k_{N}=N_{A}\left(\frac{a_{\mathrm{DPB}}}{v_{\mathrm{DPB}}}-\frac{a_{\mathrm{PI}}}{v_{\mathrm{PI}}}\right)^{2}
$$

and $S_{i}(q)$ is the structure factor for $i$ th component polymer $(i=\mathrm{DPB}$ or $\mathrm{PI})$ given by

$$
S_{i}(q)=\frac{2}{x_{i}^{2}}\left[\left(\frac{h_{i}}{h_{i}+x_{i}}\right)^{h_{i}}-1+x_{i}\right]
$$

where

$$
x_{i}=q^{2} N_{n, i} b_{i}^{2} / 6,
$$

and

$$
h_{i}=\left[\left(N_{w, i} / N_{n, i}\right)-1\right]^{-1} .
$$

$N_{n, i}$ and $N_{w, i}$ denote the number- and weight-averaged degrees of polymerization for the $i$ th component $(i=\mathrm{DPB}$ or $\mathrm{PI}$ ), respectively. $a_{i}$ and $v_{i}$ are, respectively, the scattering length and the molar volume of monomer unit, and $\phi_{i}$ the volume fraction for the $i$ th component. $N_{A}$ is Avogadro's number, $\chi$ is the Flory-Huggins interaction parameter be-

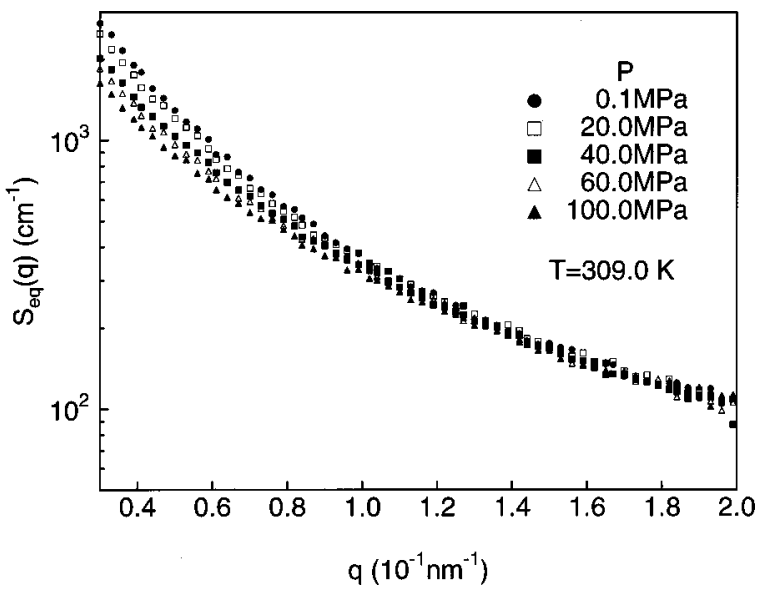

FIG. 2. Pressure dependence of the equilibrium scattering function $S_{\text {eq }}(q)$ at $309.0 \mathrm{~K}$ plotted as a function of $q$. 


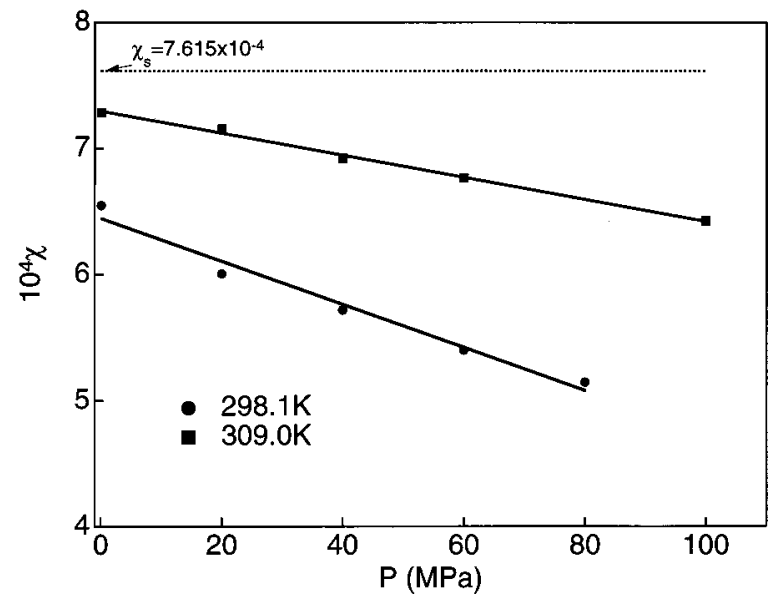

FIG. 3. Estimated $\chi$ parameters plotted as a function of pressure $P$ at 298.1 K (circles) and 309.0 K (squares).

tween DPB and PI per monomer unit, and $v_{0}$ is the reference cell volume defined as $v_{0}=\left(\phi_{\mathrm{DPB}} / v_{\mathrm{DPB}}+\phi_{\mathrm{PI}} / v_{\mathrm{PI}}\right)^{-1}$.

We fitted SANS profiles with Eq. (3) with $\chi$ and $b_{i}$ being adjustable parameters. Figure 3 shows the pressure dependence of $\chi$ parameter thus evaluated at 298.1 and $309.0 \mathrm{~K}$. The pressure dependence of $\chi$ is given by

$$
\chi=6.44 \times 10^{-4}-1.71 \times 10^{-6} P(\mathrm{MPa}) \text { at } 298.1 \mathrm{~K}(8)
$$

and

$$
\chi=7.29 \times 10^{-4}-8.74 \times 10^{-7} P(\mathrm{MPa}) \text { at } 309.0 \mathrm{~K}(9)
$$

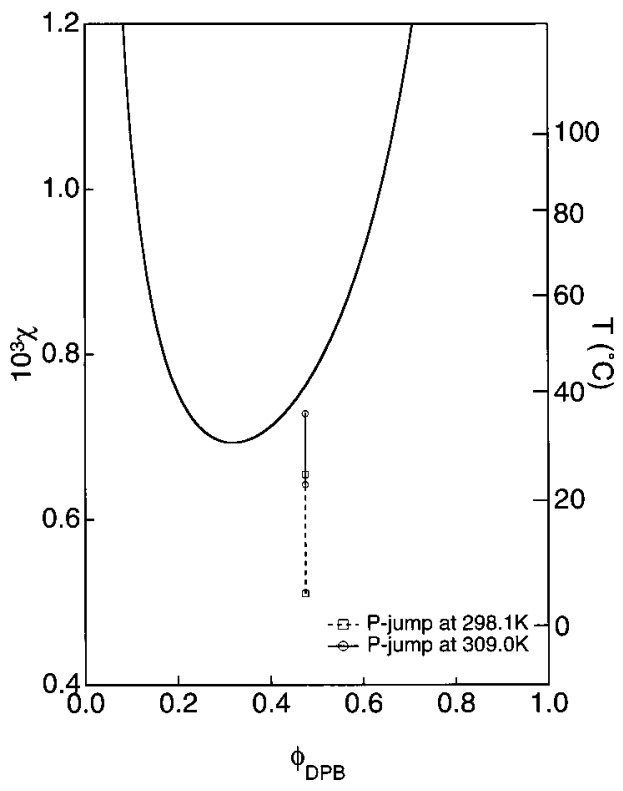

FIG. 4. Phase diagram of DPB/PI blend in the parameter space of $\chi$ (or $T$ at $0.1 \mathrm{MPa}$ ) and volume fraction of DPB in the blend of DPB/PI. Solid line indicates the spinodal line of DPB/PI blend calculated by the Flory-Huggins theory. Broken line with squares and solid line with circles represent the quench depth in $\chi$ due to the pressure jump at $298.1 \mathrm{~K}$ and $309.0 \mathrm{~K}$, respectively.

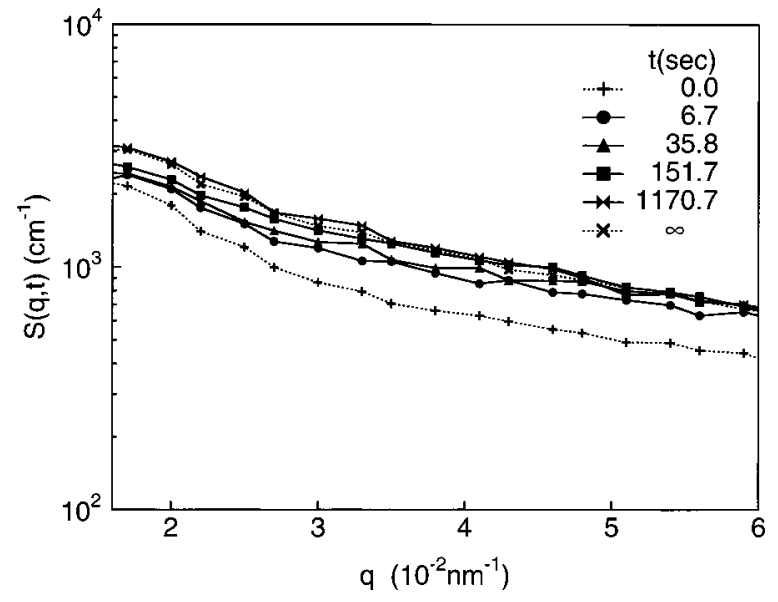

FIG. 5. Change in SANS scattering function $S(q, t)$ with time after the pressure jump from 80.0 to $0.1 \mathrm{MPa}$ at $298.1 \mathrm{~K}$.

The $\chi$ decreases with pressure, indicating that the DPB/PI mixture has the upper-critical-solution-pressure-type phase diagram. Figure 4 shows the spinodal line (solid line) plotted as a function of $\phi_{\mathrm{DPB}}$ for the DPB/PI mixture. The spinodal line was calculated with

$$
\chi_{s}=\frac{v_{0}}{2}\left[\frac{1}{\phi_{\mathrm{DPB}} v_{\mathrm{DPB}} N_{w, \mathrm{DPB}}}+\frac{1}{\phi_{\mathrm{PI}} v_{\mathrm{PI}} N_{w, \mathrm{PI}}}\right] .
$$

The ordinate axis on the right-hand side of the figure corresponds to temperature at $0.1 \mathrm{MPa}$ calculated from the following temperature dependence of $\chi$ at $0.1 \mathrm{MPa}$ that in turn was measured from SANS experiments on the same blend in the single-phase state at $0.1 \mathrm{MPa}$ as a function of temperature:

$$
\chi=2.69 \times 10^{-4}-0.606 / T \text { at } 0.1 \mathrm{MPa} .
$$

The figure also includes changes in a thermodynamic state of the blend induced by the pressure jump from 80.0 to $0.1 \mathrm{MPa}$ (squares and broken line) and 100.0 to $0.1 \mathrm{MPa}$ at $309.0 \mathrm{~K}$ (circles and solid line) which are estimated from Eqs. (8) and (9), respectively. The pressure jumps at $289.1 \mathrm{~K}$ and 309.0 $\mathrm{K}$, respectively, corresponds to the jump in the $\chi$ value $\Delta \chi$ $=1.37 \times 10^{-4}$ and $8.73 \times 10^{-5}$ or the temperature jump $\Delta T$ $=20.1 \mathrm{~K}$ and $13.0 \mathrm{~K}$ from Eqs. (8), (9), and (11).

\section{B. Changes in structure factors with time after pressure change}

Figures 5 and 6 show the changes in the scattered intensity of the DPB/PI mixtures with time after the onset of the quench from 80.0 to $0.1 \mathrm{MPa}$ at $298.1 \mathrm{~K}$ and from 100.0 to $0.1 \mathrm{MPa}$ at $309.0 \mathrm{~K}$, respectively. In both figures, after the quench, the scattering function $S(q, t)$ increases with time $t$ at observed $q$ region toward the equilibrium scattering function $S(q, \infty)$ at $0.1 \mathrm{MPa}$. The slower growth rate of the intensity is found to be at lower $q$ region.

The dynamics of concentration fluctuations in $A / B$ binary mixtures is described by time-dependent Ginzburg-Landau theory. The time-evolution of $q$-Fourier modes $\delta \phi_{A}(q, t)$ for the local concentration fluctuations of component $A$ is given by $[31-33]$ : 


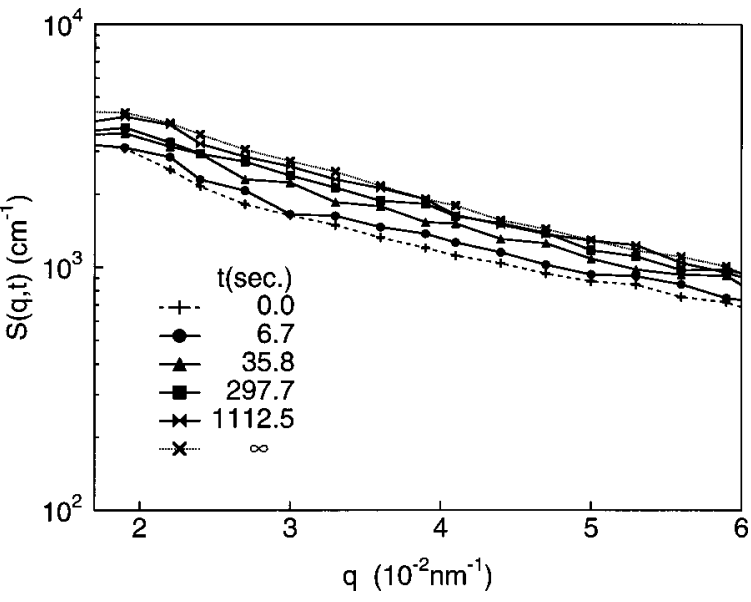

FIG. 6. Change in SANS scattering function $S(q, t)$ with time after the pressure jump from 100.0 to $0.1 \mathrm{MPa}$ at $309.0 \mathrm{~K}$.

$$
\frac{\partial}{\partial t} \delta \phi_{A}(q, t)=-\Lambda(q) q^{2} \mu(q, t)+\varsigma(q, t),
$$

where $\Lambda(q)$ is the Onsager kinetic coefficient, $\mu(q, t)$ is the local chemical potential, and $s(q, t)$ is the random thermal force term as expressed by the following fluctuationdissipation relation $[18,34]$ :

$$
\left\langle\varsigma(q, t) \varsigma\left(q^{\prime}, t^{\prime}\right)\right\rangle=-2 k_{B} T \Lambda(q) q^{2} \delta\left(t-t^{\prime}\right),
$$

where $k_{B}$ and $T$ are, respectively, the Boltzman's constant and absolute temperature, and \langle\rangle denotes thermal average. If $\delta \phi_{A}(q, t)$ is small, Eq. (12) can be linearized in terms of $\delta \phi_{A}(q, t)$ :

$$
\frac{\partial}{\partial t} \delta \phi_{A}(q, t)=-\frac{\Lambda(q) q^{2}}{\left[S(q, \infty) /\left(k_{B} T k_{N}\right)\right]} \delta \phi_{A}(q, t)+\varsigma(q, t),
$$

where $S(q, \infty) / k_{N}$ is the structure factor corresponding to the scattering function $S(q, \infty)$ is equal to $S_{\mathrm{eq}}(q)$ in Eq. (3).

We can solve Eq. (14) and hence predict time evolution of the scattering function $S(q, t)\left[\propto\left\langle\left|\delta \phi_{A}(q, t)\right|^{2}\right\rangle\right][24,25]$.

$$
S(q, t)=S(q, \infty)+[S(q, 0)-S(q, \infty)] \exp [-2 R(q) t],
$$

where $S(q, 0)$ and $R(q)$ are, respectively, $S(q, t)$ at $t=0$ and the relaxation rate of the $q$-Fourier modes of the concentration fluctuations. $R(q)$ is expressed by

$$
R(q)=q^{2} \frac{\Lambda(q)}{\left[S(q, \infty) /\left(k_{B} T k_{N}\right)\right]} .
$$

Rearranging Eq. (15), we obtain

$$
\ln [S(q, \infty)-S(q, t)]=\ln [S(q, \infty)-S(q, 0)]-2 R(q) t .
$$

Thus we can estimate $R(q)$ from the slope of $\ln [S(q, \infty)$ $-S(q, t)]$ vs $t$ plot where $\ln$ here denotes natural logarithms. In Figs. 7 and $8, \ln [S(q, \infty)-S(q, t)]$ is plotted as a function of

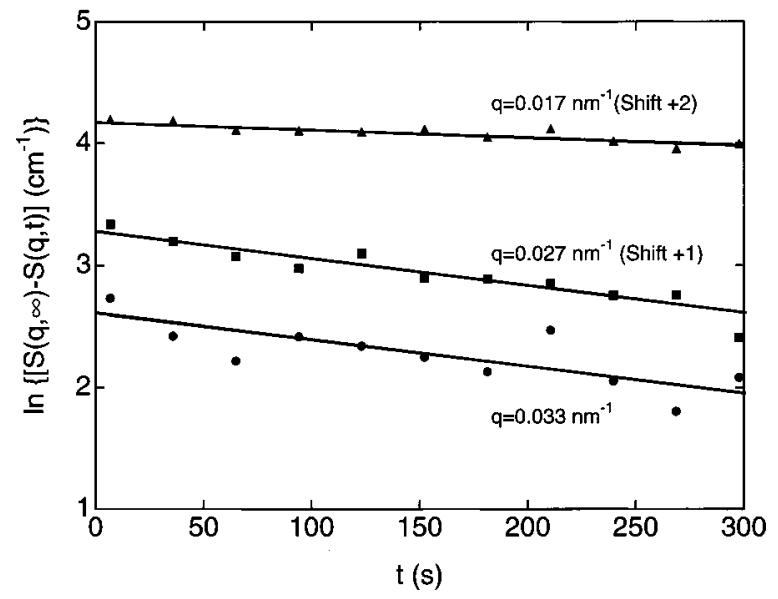

FIG. 7. $\ln [S(q, \infty)-S(q, t)]$ plotted as function of time $t$ at 298.1 $\mathrm{K}$ and at fixed $q$ values. Solid lines were obtained by linear regression of data.

$t$ after the onset of quench at 298.1 and $309.0 \mathrm{~K}$, respectively. Though the data is scattered, the linearity between $\ln [S(q, \infty)-S(q, t)]$ and $t$ can be found in each plot.

Figure 9 shows the $q$ dependence of $R(q)$ estimated from the plots of $\ln [S(q, \infty)-S(q, t)]$ vs $t$. In both experiments, the $R(q)$ increases with $q$. The values of $R(q)$ at $309.0 \mathrm{~K}$ [Fig. 9(b)] are comparable with those at $298.1 \mathrm{~K}$ [Fig. 9(a)] in the observed $q$ region, which seems to indicate that the critical slowing down is not clearly observed. This may be primarily because $309.0 \mathrm{~K}$ is not yet close enough to $314.2 \mathrm{~K}$ (spinodal temperature) and also because the increase in the Onsager kinetic coefficient with temperature is dominant comparable with the increase in $[S(q, \infty)]$ with temperature [see Eq. (16)].

$S(q, \infty)$ can be approximated by the following OrnsteinZernike-Debye form [35] at $q R_{g} \leqslant 1$ :

$$
S(q, \infty) \cong \frac{S(0, \infty)}{1+q^{2} \xi_{\mathrm{th}}^{2}}
$$

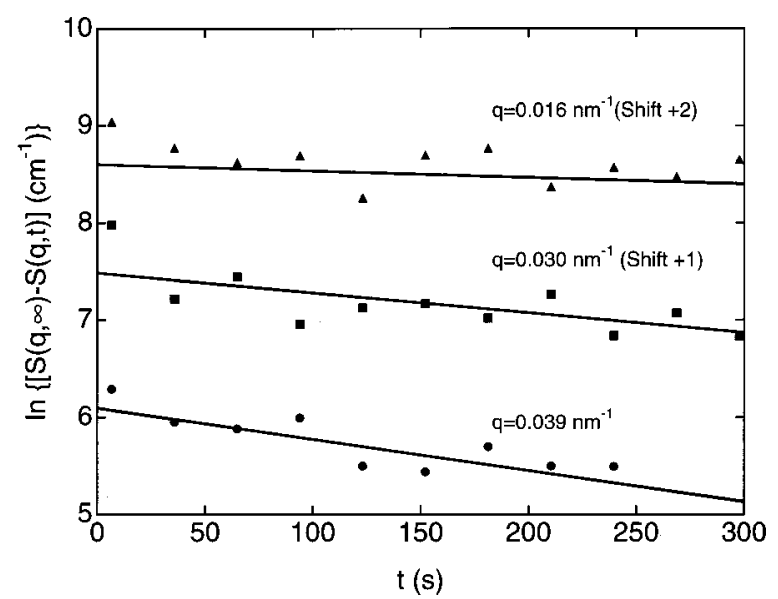

FIG. 8. $\ln [S(q, \infty)-S(q, t)]$ plotted as function of time $t$ at 309.0 $\mathrm{K}$ and at fixed $q$ values. Solid lines were obtained by linear regression of data. 


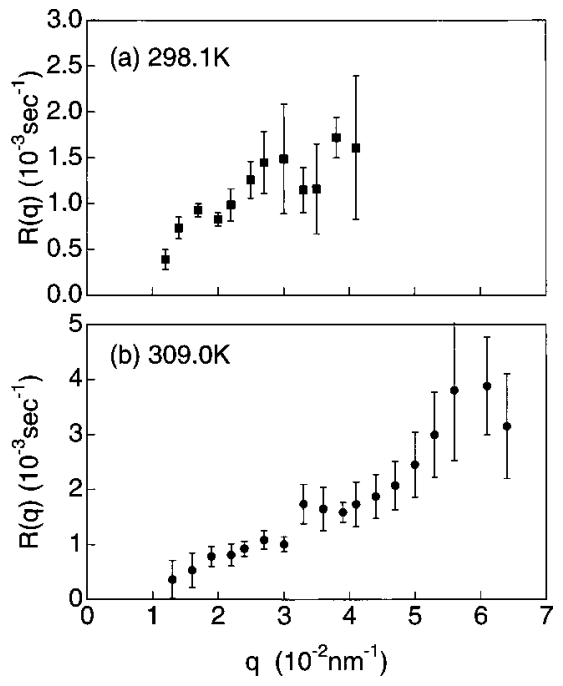

FIG. 9. Relaxation rate $R(q)$ for the $q$-Fourier mode of the concentration fluctuations plotted as a function of $q$ at (a) $298.1 \mathrm{~K}$ and (b) $309.0 \mathrm{~K}$.

where $\xi_{\text {th }}$ is the correlation length of the concentration fluctuations and given by

$$
\xi_{\mathrm{th}}^{2}=\frac{v_{0}}{36\left(\chi-\chi_{s}\right)}\left[\frac{b_{\mathrm{DPB}}^{2} N_{z, \mathrm{DPB}}}{v_{\mathrm{DPB}} \phi_{\mathrm{DPB}} N_{w, \mathrm{DPB}}}+\frac{b_{\mathrm{PI}}^{2} N_{z, \mathrm{PI}}}{v_{\mathrm{PI}} \phi_{\mathrm{PI}} N_{w, \mathrm{PI}}}\right]
$$

with $N_{z, i}$ being $z$-averaged degrees of polymerization for the $i$ th component. From Eqs. (16) and (18), we thus obtain

$$
R(q)=q^{2} \frac{\Lambda(q)\left(1+q^{2} \xi_{\mathrm{th}}^{2}\right)}{\left[S(0, \infty) /\left(k_{B} T k_{N}\right)\right]}
$$

where $\xi_{\text {th }}=1.52 \times 10^{2}$ and $2.73 \times 10^{2} \mathrm{~nm}$ at 298.1 and 309.0 $\mathrm{K}$, respectively. In the limit of $q R_{g} \rightarrow 0, \Lambda(q)$ asymptotically increases to a $q$-independent value $\Lambda(0)$. In this limit, we should have a linear relationship between $R(q) / q^{2}$ vs $q^{2}$ with a positive slope. In reality, as shown in Fig. 10, $R(q) / q^{2}$ vs $q^{2}$ decreases nonlinearly with $q^{2}$, i.e., the values $R(q) / q^{2}$ (shown by circles and squares) are much suppressed than those expected from the linear relationship [as shown by the solid lines with $\Lambda(q)=\Lambda(0)]$ even at $q^{2}$ values much smaller than $1 / R_{g}{ }^{2}, 2.27 \times 10^{-3} \mathrm{~nm}^{-2}$ for DPB and 1.11 $\times 10^{-2} \mathrm{~nm}^{-2}$ for PI. This means that the Onsager kinetic coefficient must have a $q$ dependence for some reasons even at $q R_{g} \leqslant 1$. We shall discuss the $q$ dependence of Onsager kinetic coefficient in the following section and the broken lines predicted by the Pincus theory in Sec. III D.

\section{C. $Q$ dependence of Onsager kinetic coefficient}

Figure 11 shows the $q$ dependence of the Onsager kinetic coefficient at 298.1 (squares) and at $309.0 \mathrm{~K}$ (circles), as estimated from Eq. (16), i.e.,

$$
\Lambda(q)=\frac{R(q)\left[S(q, \infty) /\left(k_{B} T k_{N}\right)\right]}{q^{2}},
$$

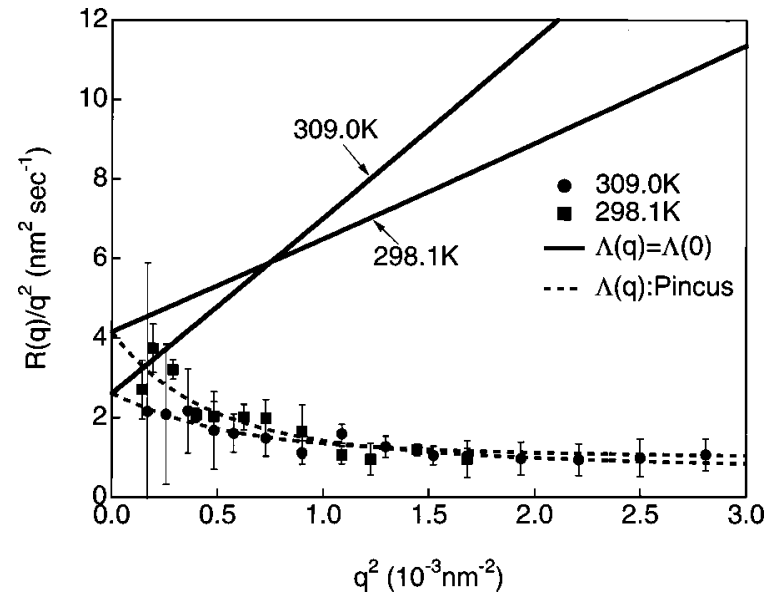

FIG. 10. $R(q) / q^{2}$ plotted against $q^{2}$ at $309.0 \mathrm{~K}$ (circles) and $298.1 \mathrm{~K}$ (squares). The solid lines are in the case where $\Lambda(q)$ $=\Lambda(0)=(5.34 \pm 0.74) \times 10^{-22}$ at $298.1 \mathrm{~K}$ and $(10.3 \pm 0.6) \times 10^{-21}$ at $309.0 \mathrm{~K}$, and the broken lines the best-fitting results given by the Pincus theory with $R_{\Lambda}$ and $\Lambda(0)$ given in Table II.

where $R(q)$ was directly measured as described in Sec. III B and shown in Fig. 9, and $S(q, \infty) / k_{N}$ is the structure factor $S_{\text {eq }}(q, \infty) / k_{N}$ at each temperature given by Eq. (3). We found that the $q^{-2}$ behavior at higher $q$ region in both quench experiments. The broken lines predicted by Pincus theory will be described in following section.

\section{Comparison between experimental and theoretical result for Onsager kinetic coefficient}

Pincus [17] derived the $q$ dependence of the Onsager kinetic coefficient for symmetric blends

$$
\Lambda(q)=\Lambda(0) \frac{1-\exp \left(-q^{2} R_{\Lambda}^{2}\right)}{q^{2} R_{\Lambda}^{2}},
$$

where the characteristic length $R_{\Lambda}$ in this theory is considered to be the radius of gyration of the symmetric polymers themselves. Although the Pincus theory cannot be applied to

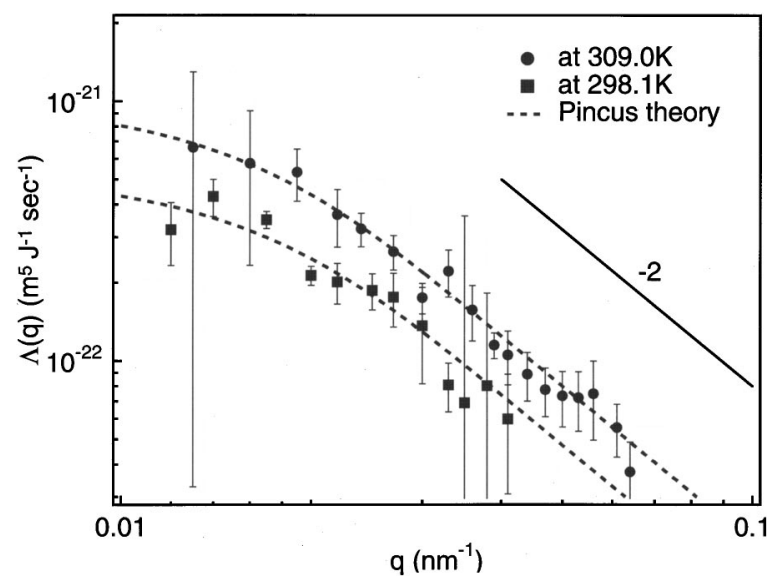

FIG. 11. $Q$ dependence of Onsager kinetic coefficient at $298.1 \mathrm{~K}$ (squares) and $309.0 \mathrm{~K}$ (circles). The broken lines indicates the bestfitting results with the Pincus theory [Eq. (22)]. 
TABLE II. Fitting parameters with Pincus theory and Doi-Onuki theory

\begin{tabular}{|c|c|c|c|c|}
\hline \multirow[b]{2}{*}{$\begin{array}{c}\text { Temperature } \\
\text { (K) }\end{array}$} & \multicolumn{2}{|c|}{ Pincus } & \multicolumn{2}{|c|}{ Doi-Onuki } \\
\hline & $\begin{array}{c}\Lambda(0) \\
\left(10^{-22} \mathrm{~m}^{5} \mathrm{~J}^{-1} \mathrm{~s}^{-1}\right)\end{array}$ & $\begin{array}{c}R_{\Lambda} \\
(\mathrm{nm})\end{array}$ & $\begin{array}{c}\Lambda(0) \\
\left(10^{-22} \mathrm{~m}^{5} \mathrm{~J}^{-1} \mathrm{~s}^{-1}\right)\end{array}$ & $\begin{array}{c}\xi_{\mathrm{ve}} \\
(\mathrm{nm})\end{array}$ \\
\hline 298.1 & $5.35 \pm 0.74$ & $67.2 \pm 8.1$ & $6.39 \pm 1.4$ & $64.6 \pm 12.6$ \\
\hline 309.0 & $10.3 \pm 0.6$ & $71.8 \pm 3.3$ & $14.1 \pm 1.7$ & $76.9 \pm 7.1$ \\
\hline
\end{tabular}

our asymmetric blends in a rigorous sense, we attempted to fit the experimental results with Eq. (22) by using $\Lambda(0)$ and $R_{\Lambda}$ as the floating parameters. The broken lines in Figs. 10 and 11 are the best-fitting results with the fitting parameters in Table II. Although Eq. (22) can well explain the experimental results on $\Lambda(q)$, the estimated values of $R_{\Lambda}$, which should be equal to $R_{g}$, are much larger than $R_{g}=21.0 \mathrm{~nm}$ for DPB and $R_{g}=9.5 \mathrm{~nm}$ for PI, which is similar to the result obtained by Schwahn, Janssen, and Springer for dPS/PVME [20]. Thus some unsolved problems are still left unveiled. By using $\Lambda(q)$ best fitted with the Pincus theory as shown by the broken lines in Fig. 11, the nonlinear behavior of $R(q) / q^{2}$ against $q^{2}$ can be apparently explained, as shown by the broken lines in Fig. 10. However, the same problem as discussed above (i.e., $R_{\Lambda} \gg R_{g}$ ) still remains to be solved.

Doi and Onuki [22] took into account dynamical coupling between stress and diffusion for dynamically asymmetric blends and derived the $q$ dependence of the Onsager kinetic coefficient

$$
\Lambda(q)=\frac{\Lambda(0)}{1+q^{2} \xi_{\mathrm{ve}}^{2}}
$$

with the viscoelastic length $\xi_{\mathrm{ve}}$ defined by

$$
\xi_{\mathrm{ve}}=\left(\frac{4}{3} \alpha \eta \Lambda(0)\right)^{1 / 2},
$$

where $\eta$ is the zero shear viscosity of the mixture and $\Lambda(0)$ is expressed by

$$
\Lambda(0)=\phi_{A} \phi_{B}\left(D_{A} N_{A} \phi_{B}+D_{B} N_{B} \phi_{A}\right) v_{0} / k_{B} T
$$

and $\alpha$ is a dynamical asymmetry parameter defined by

$$
\alpha=\frac{D_{A} N_{A}-D_{B} N_{B}}{D_{A} N_{A} \phi_{B}+D_{B} N_{B} \phi_{A}} .
$$

Here $D_{i}$ and $N_{i}$ are, respectively, the self-diffusion coefficient and the polymerization index of $i$ th component $(i=A$ or $B$ in this case) in the blend.

We fitted the experimental results with Eq. (23) by using $\Lambda(0)$ and $\xi_{\mathrm{ve}}$ as adjustable parameters. The best-fitting results are displayed by the solid lines in Fig. 12 together with the theoretical predictions based on the Pincus theory with $R_{\Lambda}$ $=R_{g}=9.5 \mathrm{~nm}$ for PI and $21.0 \mathrm{~nm}$ for DPB. The DO theory can well predict the experimental results on $\Lambda(q)$. The estimated $\Lambda(0)$ and $\xi_{\mathrm{ve}}$ are also listed in Table II. $\xi_{\mathrm{ve}}$ is much larger than $R_{g}$ of DPB and PI, revealing that the viscoelastic effects play a dominant role on the observed $q$ dependence of the Onsager kinetic coefficient. The effects suppress the transport coefficient even at $q R_{g} \ll 1$ in the range of $q$ satisfying $q \xi_{\mathrm{ve}} \geqslant 1$, as discussed in detail elsewhere [36]. The effects are screened out at $q \xi_{\mathrm{ve}}<1$.

In contrast, the Pincus theory predicts the suppression of the transport coefficient occurs only when $q \geqslant 1 / R_{g}$. This is natural because, when the component polymers are symmetric, the dynamical asymmetry parameters $\alpha$ goes to zero and hence $\xi_{\mathrm{ve}}$ goes to zero, resulting in a complete screening of the viscoelastic effects. Thus the suppression of the transport coefficient occurs only through the $q$ dependence of the reptation modes. In other words physical origin of the suppression is completely different. In our asymmetric blends, the suppression of $\Lambda(q)$ at $1 / \xi_{\mathrm{ve}}<q<1 / R_{g}$ reflects the viscoelastic effects and that at $q \geqslant 1 / R_{g}$ would be affected by the $q$ dependence of the reptation modes as well. Figure 13 highlights the effects of $\Lambda(q)$ on the plot of $R(q) / q^{2}$. The figure clearly reveals that two kinds of the suppression on $\Lambda(q)$ affect the $q$ dependence of the growth rate $R(q)$ in a different $q$ range.

\section{E. Comparison of $\xi_{\mathrm{ve}}$ estimated from the time-resolved SANS with $\xi_{\mathrm{ve}}$ evaluated from an independent experiment}

Let us now compare the value of $\xi_{\mathrm{ve}}$ experimentally estimated from $\Lambda(q)$ based on Eq. (23) and the SANS relaxation experiments with that estimated from independent ex-

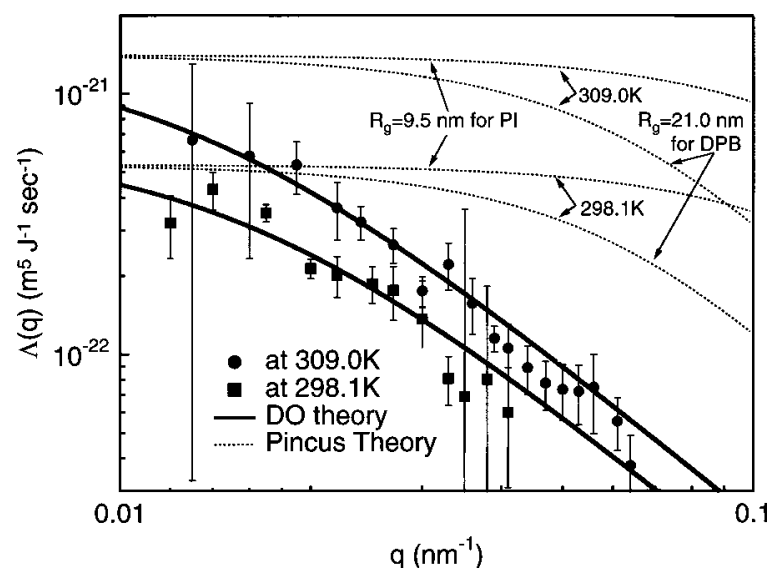

FIG. 12. $Q$ dependence of Onsager kinetic coefficient at $309.0 \mathrm{~K}$ (circles) and $298.1 \mathrm{~K}$ (squares). The solid lines indicate the fitting results with the DO theory [Eq. (23)] with $\Lambda(0)$ and $\xi_{\text {ve }}$ given in Table II, while the broken lines indicate the prediction given by the Pincus theory for the symmetric blends, with $\Lambda(0)$ given in Table II and $R_{g}=9.5$ and $21 \mathrm{~nm}$, free from the viscoelastic effects. 


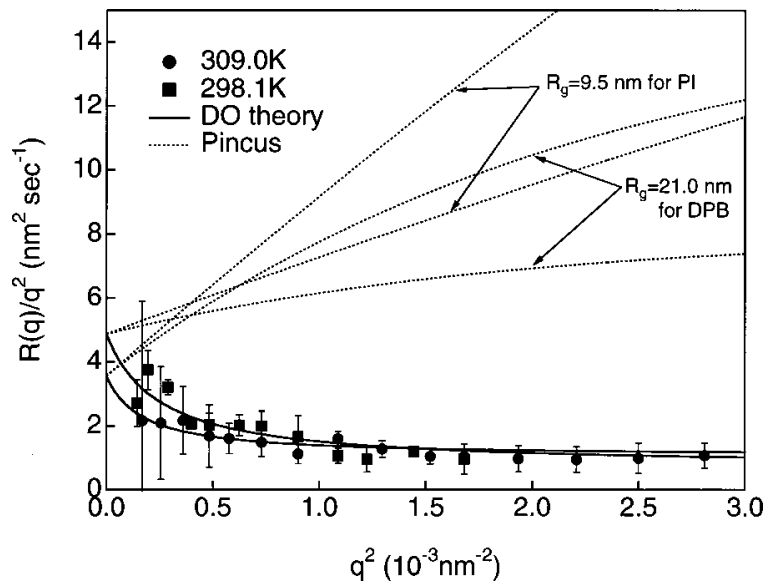

FIG. 13. $R(q) / q^{2}$ plotted against $q^{2}$ at $309.0 \mathrm{~K}$ (circles) and $298.1 \mathrm{~K}$ (squares). The solid lines are the best-fitting results with the DO theory with $\Lambda(0)$ and $\xi_{\text {ve }}$ given in Table II, while the broken lines are the results predicted by the Pincus theory for the symmetric blends, with $\Lambda(0)$ given in Table II and $R_{g}=9.5$ and $21 \mathrm{~nm}$, free from the viscoelastic effects.

periments based on Eq. (24). The independent experiments should lead to estimate the values of $\eta, \Lambda(0)$, and $\alpha$. We obtained $\eta=6.96 \times 10^{6} \mathrm{Pas}$ at $298.1 \mathrm{~K}$ from the dynamic viscoelastic measurements as a function of $\omega$. We further need the self-diffusion coefficients of DPB and PI in the blends to estimate $\alpha$ and $\Lambda(0)$. Unfortunately, however, we do not have the data of the self-diffusion coefficient of each component. Thus, we estimated them from the dynamic viscoelastic measurement of the blend at $298.1 \mathrm{~K}$ in this work.

Figure 14 shows the frequency dependence of the loss modulus $G^{\prime \prime}(\omega)$ of the DPB/PI blend at $298.0 \mathrm{~K}$. The curve of $G^{\prime \prime}(\omega)$ has one peak at $\omega=4.0 \mathrm{~s}^{-1}$ and one shoulder at $\omega=0.02 \mathrm{~s}^{-1}$ as indicated by the arrows in the figure, indicating that there are two relaxation processes in the blend. The fast relaxation process, associated with the maximum at 4.0 $\mathrm{s}^{-1}$, corresponds to the relaxation of the PI component, while the slow relaxation process, associated with the shoulder at $0.02 \mathrm{~s}^{-1}$, corresponds to the DPB component. Struglinski

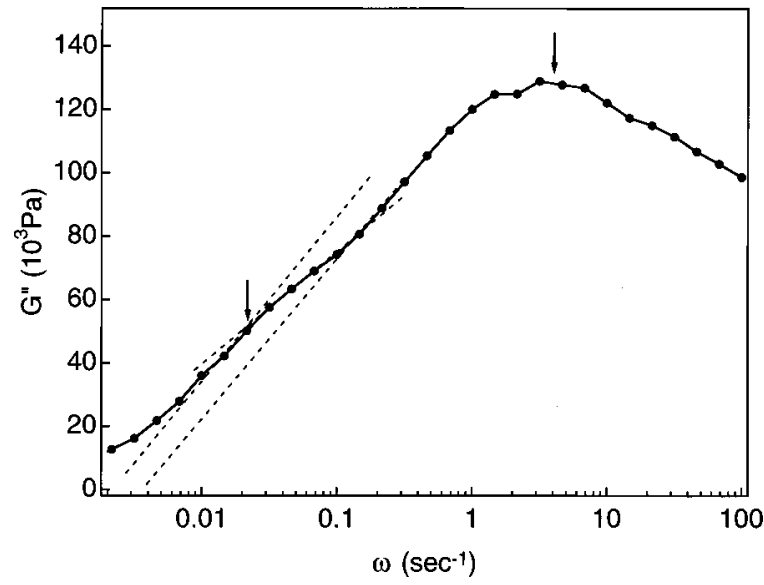

FIG. 14. Frequency dependence of shear loss modulus $G^{\prime \prime}(\omega)$ for DPB/PI blend at $298.0 \mathrm{~K}$. The arrows indicate the peak or shoulder in $G^{\prime \prime}(\omega)$. and Graessley [37] measured the viscoelastic properties for polybutadiene. They found the angular frequency $\omega_{\max }$ at the maximum of $G^{\prime \prime}$ has the following relationship with the viscoelastic characteristic time $\tau_{0}$,

$$
\omega_{\max }^{-1}=0.45 \tau_{0}
$$

where $\tau_{0}$ is defined by

$$
\tau_{0}=\eta J_{e}^{0}
$$

with $J_{e}^{0}$ being steady-state compliance. Here this relationship is assumed to be valid for the viscoelastic behavior of DPB and PI in the blend. The $\tau_{0}$ value estimated for DPB and PI in the blends are, respectively, $0.02^{-1} / 0.45=1.1 \times 10^{2}$ and $4.0^{-1} / 0.45=5.6 \times 10^{-1} \mathrm{~s}$ at $298.0 \mathrm{~K}$.

Next, we need to know the relationship between $\tau_{0}$ and the characteristic time $\tau_{d, i}$ for self-diffusion defined by

$$
\tau_{d, i}=R_{g, i}^{2} / D_{s, i} \quad(i=\mathrm{DPB} \text { or } \mathrm{PI}) .
$$

Pearson, Fetters, and Graessley [38] measured the viscosity and the self-diffusion coefficient of hydrogenated polybutadiene (HPB) with varying molecular weight. From their data, we can estimate the ratio $\tau_{d, i} / \tau_{0, i}(i=\mathrm{DPB}$ or PI) as a function of molecular weight. We used $\tau_{d, i} / \tau_{0, i}=5.77$, which is obtained for HPB with $M_{w}=1.0 \times 10^{5}$, for the estimation of $\tau_{d, i}$. From the estimated $\tau_{d, i}$, and $R_{g, i}$, we obtained the self-diffusivity for DPB $\left(D_{\mathrm{DPB}}\right)$ and PI $\left(D_{\mathrm{PI}}\right)$ from Eq. (27): $D_{\mathrm{DPB}}=8.9 \times 10^{-19} \mathrm{~m}^{2} / \mathrm{sec}$ and $D_{\mathrm{PI}}=3.1 \times 10^{-17} \mathrm{~m}^{2} / \mathrm{sec}$ at $298.0 \mathrm{~K}$.

Substituting the estimated self-diffusivity of each component into Eqs. (25) and (26), we obtained corresponding quantities that are defined hereafter $\Lambda(0)_{\text {cal }}$ and $\alpha_{\text {cal }}$ : $\Lambda(0)_{\mathrm{cal}}=1.7 \times 10^{-22} \mathrm{~m}^{5} / \mathrm{J} \mathrm{s}$ and $\alpha_{\mathrm{cal}}=1.37$ at $298.0 \mathrm{~K}$. The ratio $\Lambda(0)_{\exp } / \Lambda(0)_{\text {cal }}$ is 3.7 , which shows a fair agreement, though not perfect, in view of the assumption and estimation errors involved. Here $\Lambda(0)_{\exp }$ is the value $\Lambda(0)$ estimated from the DO theory by using Eq. (23). Finally, we estimated the viscoelastic length from Eq. (24), which we defined as $\xi_{\mathrm{ve}, \mathrm{cal}}=5.4 \times 10 \mathrm{~nm}$ at $298.0 \mathrm{~K}$. The ratio $\xi_{\mathrm{ve}, \exp } / \xi_{\mathrm{ve}, \mathrm{cal}}$ is 1.2 , indicating that the DO theory well explains our experimental result. This suggests that the viscoelastic effect on the $q$ dependence of the Onsager kinetic coefficient dominates over the effect of the normal modes on the $q$ dependence of the Onsager coefficient in the $q$ range and time scale of our observation for this blend. The slight discrepancy between the theoretical and experimental results may originate from errors involved by the estimation of the self-diffusion coefficients from the viscoelastic measurements. In order to avoid this uncertainty, we need to measure the tracer diffusion coefficient of each component by using forced Rayleigh scattering technique [39].

\section{CONCLUSION}

We measured relaxation processes of the concentration fluctuations in a single-phase state induced by rapid pressure change for an asymmetric polymer blend DPB/PI by using time-resolved small-angle neutron scattering. The changes in 
the scattered intensity with time during the relaxation processes were found to be approximated by the Cahn-HilliardCook linearized theory. The CHC analysis yielded the $q$ dependence of the Onsager kinetic coefficient $\Lambda(q) \equiv \Lambda(0)$ (1 $\left.+q^{2} L_{c}^{2}\right)^{-1}$, which has the $q^{-2}$ dependence at $q L_{c}>1$ with $L_{c}$ being the experimentally assessed characteristic length. The $L_{c}$ value thus evaluated was much larger than the radii of gyration of DPB and PI, inconsistent with the Pincus theory. This inconsistency is believed to be reasonable, because the Pincus theory is developed for the purely symmetric blends where the stress-diffusion coupling and hence the viscoelastic effects on the transport coefficient as elucidated by Doi and Onuki theory are absent. Thus the application of the theory to the asymmetric blends itself is problematic for a rigorous and quantitative analysis. The $L_{c}$ value is 1.2 times $\xi_{\mathrm{ve}}$ that is predicted by Doi and Onuki theory together with the fundamental parameters as obtained from viscoelastic data, indicating that the viscoelastic effects arising from dynamical asymmetry between DPB and PI give rise to the $q$ dependence of $\Lambda(q)$ in the length scale of our observation, which is much larger than the radius of gyration $R_{g}$ or in the $q$ range of our observation that is much smaller than $1 / R_{g}$.

\section{ACKNOWLEDGMENTS}

The authors are grateful to Professor H. Watanabe, Institute for Chemical Research, Kyoto University, for valuable discussion on the viscoelastic measurement for evaluation of the self-diffusivity. This work was supported in part by a Grant-in-Aid from Japan Society for the Promotion of Science (Nos. 12640392 and 13031053) and by the Sumitomo Foundation.
[1] T. Hashimoto, Phase Transitions 12, 47 (1988).

[2] T. Hashimoto, in Structure and Properties of Polymers, Material Science and Technology Series, edited by E. L. Thomas (VCH, Weinheim, 1993), p. 252.

[3] T. Hashimoto, H. Jinnai, Y. Nishikawa, T. Koga, and M. Takenaka, Prog. Colloid Polym. Sci. 106, 118 (1997).

[4] T. Hashimoto, T. Koga, H. Jinnai, and Y. Nishikawa, Nuovo Cimento Soc. Ital. Fis., D 20D, 1947 (1998).

[5] K. Binder and D. Stauffer, Phys. Rev. Lett. 33, 1006 (1974).

[6] K. Binder, Phys. Rev. B 15, 4425 (1977).

[7] H. Furukawa, Adv. Phys. 34, 703 (1985).

[8] J. S. Langer, M. Bar-on, and H. D. Miller, Phys. Rev. A 11, 1417 (1975).

[9] Y. Chou and W. I. Goldburg, Phys. Rev. A 20, 2105 (1979).

[10] P. E. Rouse, J. Chem. Phys. 21, 1272 (1953).

[11] B. H. Zimm, J. Chem. Phys. 24, 269 (1956).

[12] A. Z. Akcasu, Macromolecules 22, 3682 (1989).

[13] G. R. Strobl, Macromolecules 18, 558 (1985).

[14] G. Müller, D. Schwahn, H. Eckerlebe, J. Rieger, and T. Springer, J. Chem. Phys. 104, 5326 (1996).

[15] M. Doi and S. F. Edwards, The Theory of Polymer Dynamics (Clarendon, London, 1986).

[16] P. G. de Gennes, J. Chem. Phys. 72, 4756 (1980).

[17] P. Pincus, J. Chem. Phys. 75, 1996 (1981).

[18] K. Binder, J. Chem. Phys. 79, 6387 (1983).

[19] H. Jinnai, H. Hasegawa, T. Hashimoto, and C. C. Han, J. Chem. Phys. 99, 8154 (1993).

[20] D. Schwahn, S. Janssen, and T. Springer, J. Chem. Phys. 97, 8775 (1992).

[21] K. Kawasaki and T. Koga, Physica A 201, 115 (1993).

[22] M. Doi and A. Onuki, J. Phys. II (France) 2, 1631 (1992).
[23] A. Onuki and T. Taniguchi, J. Chem. Phys. 106, 5761 (1997).

[24] J. W. Cahn, J. Chem. Phys. 42, 93 (1965).

[25] H. E. Cook, Acta. Met. 18, 297 (1970).

[26] H. Takeno, M. Nagao, Y. Nakayama, H. Hasegawa, T. Hashimoto, H. Seto, and M. Imai, Polym. J. (Tokyo) 29, 931 (1997).

[27] H. Hasegawa, N. Sakamoto, H. Takeno, H. Jinnai, T. Hashimoto, D. Schwahn, H. Frielinghaus, S. Janßsen, M. Imai, and K. Mortensen, J. Phys. Chem. Solids 60, 1307 (1998).

[28] P. G. de Gennes, Scaing Concepts in Polymer Physics (Cornell University, Ithaca, 1979).

[29] S. Sakurai, T. Izumitani, H. Hasegawa, T. Hashimoto, and C. C. Han, Macromolecules 24, 4844 (1991).

[30] S. Sakurai, K. Mori, A. Okawara, K. Kimishima, and T. Hashimoto, Macromolecules 25, 2679 (1992).

[31] K. Kawasaki, Prog. Theor. Phys. 57, 826 (1977).

[32] K. Kawasaki and T. Ohta, Prog. Theor. Phys. 59, 362 (1978).

[33] T. Koga and K. Kawasaki, Phys. Rev. A 44, R817 (1991).

[34] J. D. Gunton, M. S. Miguel, and P. S. Sahni, Phase Transitions 8, 269 (1983).

[35] L. S. Ornstein and F. Zernike, Proc. R. Acad. Sci. Amsterdam 17, 793 (1914).

[36] N. Toyoda, M. Takenaka, S. Saito, and T. Hashimoto, Polymer 42, 9193 (2001).

[37] M. Struglinski and W. Graessley, Macromolecules 18, 2630 (1985).

[38] D. Pearson, L. Fetters, and W. Graessley, Macromolecules 27, 711 (1994).

[39] M. Antonietti, J. Coutandin, and H. Sillescu, Macromolecules 19, 793 (1986).

[40] J. Mays, N. Hadjichristidis, and L. J. Fetters, Macromolecules 17, 2723 (1984). 